\title{
The Risk of Posttraumatic Stress Disorder After Trauma Depends on Traumatic Load and the Catechol-O-Methyltransferase Val ${ }^{158}$ Met Polymorphism
}

\author{
Iris-Tatjana Kolassa, Stephan Kolassa, Verena Ertl, Andreas Papassotiropoulos, and \\ Dominique J.-F. De Quervain
}

\begin{abstract}
Background: The risk for posttraumatic stress disorder (PTSD) depends on the number of traumatic event types experienced in a dose-response relationship, but genetic factors are known to also influence the risk of PTSD. The catechol-O-methyltransferase (COMT) Val158Met polymorphism has been found to affect fear extinction and might play a role in the etiology of anxiety disorders.
\end{abstract}

Methods: Traumatic load and lifetime and current diagnosis of PTSD and COMT genotype were assessed in a sample of 424 survivors of the Rwandan Genocide living in the Nakivale refugee camp in southwestern Uganda.

Results: Higher numbers of different lifetime traumatic event types led to a higher prevalence of lifetime PTSD in a dose-response relationship. However, this effect was modulated by the COMT genotype: whereas Val aliele carriers showed the typical dose-response relationship, Met/Met homozygotes exhibited a high risk for PTSD independently of the severity of traumatic load.

Conclusions: The present findings indicate a gene-environment interaction between the human COMT Val158Met polymorphism and the number of traumatic event types experienced in the risk of developing PTSD.

Key Words: COMT polymorphism, genetic polymorphisms, posttraumatic stress disorder, refugees, risk

$\mathrm{P}$ ostraumatic stress disorder (PTSD) is a frequent consequence of severe traumatic experiences such as torture, war, and genocide (1). Prevalence rates of PTSD of $30 \%-40 \%$ have been reported in survivors of civil war (2). The risk of P'TSD depends on the number of different traumatic event types experienced (subsequently called "traumatic load"), with the probability for developing PTSD approaching $100 \%$ with sufficiently high trauma load $(2-4)$.

Catechol-O-methyltransferase (COMT) is involved in the catalysis and thus inactivation of catecholamines, including dopamine, epinephrine, and norepinephrine $(5,6)$. It is encoded on chromosome $22 \mathrm{q} 11.1-\mathrm{q} 11.2$ and contains a common functional polymorphism at codon 158. A substitution of valine (Val) by methionine (Met) is associated with lower enzyme activity and consequent slower catalysis of catecholamines. The difference in activity between $\mathrm{Val} / \mathrm{Val}$ and Met/Met genotypes is three- to fourfold, with Val/Met genotypes showing an intermediate activity (7). The low-activity Met allele has been associated with: higher reactivity to unpleasant visual stimuli and lower emotional resilience against negative mood states (8); higher sensitivity to pain stress (9); lower ability to upregulate opioid release after

From Clinical PSychology and Neuropsychology (1-TK, VE); Zukunftskolleg (I-TK), University of Konstanz, Konstanz, Germany; Rese arch and Innovation (SK), Simulation, Analysis and Forecasting (SAP) AG, Tägerwilen; Division of Molecular Psychology (AP); Life Sciences Training Facility (AP), Blozentrum; Division of Cognitive Neuroscience (DJ-FDQ); and the Psychiatric University Clinic (DJ-FDQ), Unversity of Basel, Basel, Switzerland.

Address correspondence to lris-Tatjana Kolassa, Dr., Clinical Psychology and Neuropsychology, University of Konstanz, Universitätsstrasse $10,78,457$ Konstanz, Germany; E-mail: Iris.Kolassa@uni-konstanzde. pain or stress $(9,10)$; higher sensory and affective ratings of pain and a more negative internal affective state (10); diminished fear extinction (11); a potentiated startle reflex (12); higher anxiety in women (13); higher incidence of major depression (14) but also with reduced generalized anxiety across adolescence, especially in interaction with a short 5-HTTLPR allele (15); less depression in men (16); better performance in tests of frontal lobe function (for a review see [171); increased activation in the hippocampal formation and the ventrolateral prefrontal cortex in response to faces displaying negative emotion (18); improved processing efficiency in the prefrontal cortex and cingulate (8); and a lower P50 gating deficit in schizophrenia (19). However, in spite of the strong influence of genotype on COMT activity, the relationship between the $\mathrm{Val}^{158} \mathrm{Met}$ polymorphism and behavior, psychiatric disorders, and cognition has been found to be moderate $(8,16,20)$. In particular, a direct influence of the COMT Val ${ }^{158}$ Met polymorphism on the most widely studied disorders-schizophrenia, bipolar disorder, substance dependence, obsessive-compulsive disorder, anorexia nervosa, and attention-deficit/hyperactivity disorder (ADHD)-has not been conclusively shown $(17,21)$. The relationship between the COMT Val ${ }^{158} \mathrm{Met}$ polymorphism and various psychiatric disorders seems to be complex and might depend on gene-gene and/or gene-environment interactions.

Gene-environment interactions occur when the effect of exposure to an environmental pathogen on a person's health depends on his or her genotype (22) for example, when the susceptibility to develop PTSD after traumatization depends on the interaction of trauma load with a certain genotype. Geneenvironment interactions have been reported for various disorders $(4,22)$. For the COMT Val ${ }^{158}$ Met polymorphism, carriers of the Val allele have been found to be at higher risk for psychotic symptoms and schizophreniform disorder after cannabis consumption, whereas Met/Met homozygotes exhibited no such consequence of cannabis use (23). In addition, Val/Val homozy- 
gotes among ADHD sufferers are more susceptible to prenatal risk factors for early-onset antisocial behavior (24)

Although carriers of the COMT Met allele seem to be at higher risk for developing anxiety disorders, as mediated by changes in both the affective and the memory systems as detailed in the preceding text, there have been no analyses on the role of the COMT Val ${ }^{158}$ Met polymorphism in the development of PTSD (25). In particular, possible gene-environment interactions between COMT genotype and traumatic load are, so far, unclear. In light of the present knowledge about the effects of COMT' genotype, COMT gene-environment interactions, and the doseresponse effect of traumatic load, Met/Met genotype carriers were hypothesized to be at higher risk to develop PTSD even after smaller numbers of different traumatic event types experienced than Val/Met and Val/Val genotype carriers, which should manifest in a COMT gene-environment interaction on the probability of developing PTSD after cumulative traumatization.

\section{Methods and Materials}

\section{Subjects}

Four hundred twenty-four Rwandese refugees ( 226 male, 198 female; mean age $=34.8$ years, $S D=5.8$, age range $17-68$ ) from the 1994 Rwandan civil war who were living in the Nakivale refugee camp in southwestern Uganda were investigated. Because the Nakivale refugee camp has grown over the last decade and is spread over a large area, participants were sampled proportionally to the population size from each zone. To exdude genetic relatives in the samples, only one person/household was interviewed. Interviewers were trained to detect current alcohol abuse and acute psychotic symptoms; candidates exhibiting these signs were excluded. All subjects had experienced multiple highly aversive traumatic situations and were examined in 2006 2007 by trained experts with a structured interview based on the Posttraumatic Diagnostic Scale (PDS) (20) with the help of trained interpreters. Traumatic events were assessed with a checklist of 36 war-and non-war-related traumatic event types (e.g, injury by weapon, rape, accident) $(2,27)$. Traumatic load was estimated by assessing the number of different traumatic event types experienced or witnessed. This measure is considered more reliable than assessing the frequency of traumatic events (2). Depressive symptoms were assessed with the depression section of the Hopkins Symptom Checklist (HSCL-D) (28). A subset of this sample has been analyzed in previous studies $(4,27)$. The procedures were approved by the Ethics Committee of the University of Konstanz, Germany, and the Ethics Committee of the Mbarara University of Science and Technology, Uganda.

The PDS and event list were completed in the form of a standardized interview. Interviewers were first trained in a 6-week course on principles of quantitative data collection and interviewing techniques. Instruments were translated into $\mathrm{Kin}$ yarwanda with several steps of translations, blind back-translations, and subsequent corrections by independent groups of translators (29). After the translations, the psychometric properties of the translated scales were investigated in a validation study including a retest spanning a 2 -week period and a crossvalidation with expert rating (30).

of the sample, $80.2 \%$ fulfilled criteria for lifetime and $48.8 \%$ for current PTSD according to the DSM-IV (1). All but one subject had experienced at least one event fulfilling the A1- and A2criterion according to DSM-IV for a traumatic event. On average, participants had experienced 12.6 different traumatic event types $(\mathrm{SD}=5.0$, range $0-25)$.

Saliva samples were obtained from each person with Oragene DNA Self-Collection Kit (DNA Genothek, Ottawa, Ontario, Canada). The DNA was extracted from saliva with standard protocols. The COMT genotype was analyzed by Pyrosequencing. Please contact the authors for primer sequences and polymerase chain reaction conditions.

\section{Statistics}

Dependent variables (lifetime and current PTSD diagnosis as well as probability of remission from PTSD, defined as "lifetime but not current PTSD") were analyzed with logistic linear regressions with the software package R (31). Further dependent variables were current PDS symptom subscales for intrusions, avoidance, and hyperarousal as well as current HSCL-D, which were analyzed with ordinary linear regressions. We did not assess lifetime PDS symptom subscales or lifetime HSCL-D, because these symptoms cannot be reliably quantified retrospectively. The number of lifetime traumatic event types was modeled via a restricted cubic spline (32) to include possible nonlinearities in the influence of traumatic load. Three knots were used, set at the 10\%, 50\%, and $90 \%$ quantiles of the covariate distribution (Table 2.3 in [32]). Nested models were compared with likelihood ratio (LR) tests (32); statistical significance was assessed by nonparametric permutation tests, with 10,000 random permutations (33). All reported $p$ values for $L R, F$ and $\chi^{2}$ tests are one-tailed as always; for $t$ tests and Kendall's $\tau$ they are twotailed See Supplement 1 for details on the statistical procedure.

\section{Results}

Subjects ( $n=424)$ were genotyped as Met/Met $(n=46)$, Val/Met $(n=190)$, and Val/Val $(n=188)$. The genotype distributions did not deviate from Hardy-Weinberg equilibrium $\left[\chi^{2}(1)=.03, p=85\right]$. Genotype groups did not differ in number of traumatic event types experienced $[F(2,421)=1.83, p=.16]$. The Met/Met genotype carriers had experienced an average of 12.9 traumatic event types ( $S D=4.9$ ), Val/Met genotype carriers had experienced an average of $13.0(\mathrm{SD}=5.2)$, and $\mathrm{Val} / \mathrm{Val}$ genotype carriers had experienced an average of $12.1(\mathrm{SD}=4.8)$.

In addition, there were no genotype differences between genders $\left[\chi^{2}(2)=2.00, p=.37\right.$. Among Met/Met genotype carriers, there were 20 women and $26 \mathrm{men}$; among Val/Met genotype carriers, there were 83 women and $107 \mathrm{men}$; and among Val/Val genotype carriers, there were 95 women and 93 men. However, traumatic load differed between the genders $[t(396)=460, p<.0001]$, with men having experienced more traumatic event types $(M=13.63, S D=4.60)$ than women $(M=$ $11.42, \mathrm{SD}=5.20$ ).

Higher numbers of different lifetime traumatic event types led to a higher prevalence of lifetime PTSD in a dose-response relationship, $\mathrm{LR}=43.73, p<.0001$ (Figure 1). There was no main effect of genotype on lifetime PTSD, LR $=1.53, p=47$. Overall, $79.3 \%$ of $\mathrm{Val} / \mathrm{Val}$ genotype carriers, $79.5 \%$ of $\mathrm{Val} / \mathrm{Met}$ genotype carriers, and $87.0 \%$ of Met/Met genotype carriers suffered from lifetime PTSD. However, the probability of developing lifetime PTSD exhibited a gene-environment interaction (i.e., an interaction of genotype by traumatic load), $L R=10.49, p=.04$. Figure 2 shows that Met/Met genotypes were at high risk for PTSD even with small traumatic load and exhibited no correlation between traumatic load and frequency of lifetime PTSDKendall's $\tau=.06, p=.34$-whereas the risk for PTSD gradually rose with traumatic load for other genotypes, with correlations 


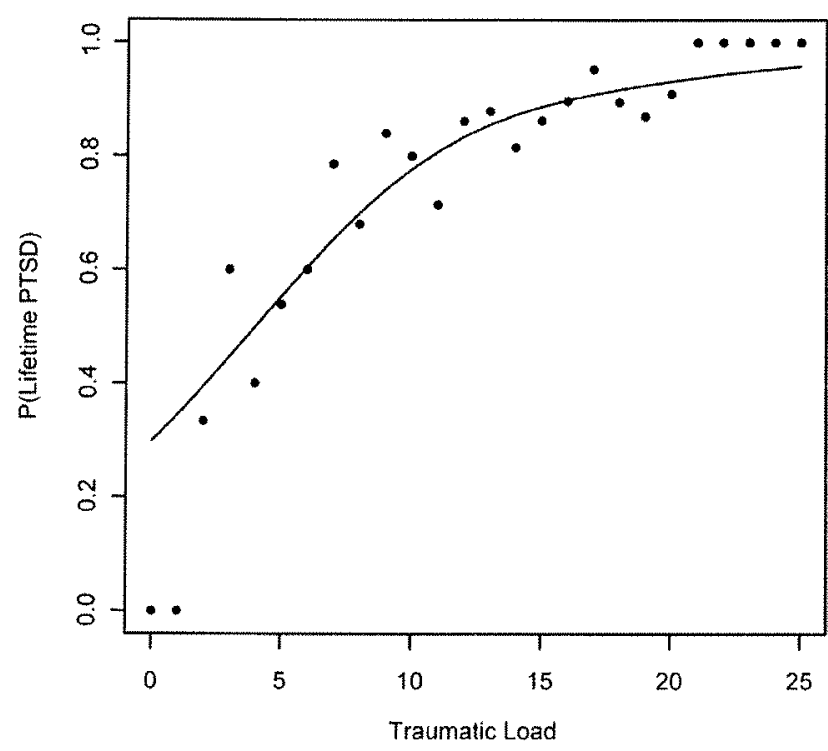

Figure 1. Observed frequencies of participants with lifetime posttraumatic stress disorder (PTSD) (dots) and model fit for lifetime PTSD probability (ine), depending on number of traumatic event types: all genotypes.

between traumatic load and frequency of lifetime PTSD of $\tau=$ $.31, p<.0001$ for $\mathrm{Val} / \mathrm{Met}$ genotypes and $\tau=.36, p<.0001$ for $\mathrm{Val}$ Val genotypes. There was little difference in PTSD prevalence between genotypes beyond 15 traumatic event types.

There was no significant main effect of gender or significant interactions of gender $X$ genotype or gender $X$ traumatic load on the probability of suffering from lifetime PTSD, all $p>46$. The probability of suffering from current PTSD, the current symptom severity as measured by the PDS, and the probability of remission from lifetime PTSD exhibited neither genotype group effects nor interactions with traumatic load, all $p>06$.

There was a significant main effect of traumatic load on HSCL-D $(L R=45.20, p<.0001)$ but no main effect of genotype (LR $=.62, p=.73)$ or a significant interaction of traumatic load $\times$ genotype $(\mathrm{LR}=6.94, p=.14)$. Similarly, there was a significant main effect of HSCL-D on lifetime PTSD probability $(\mathrm{LR}=70.05, p<.0001)$ but no main effect of genotype $(\mathrm{LR}=4.13, p=14)$ or a significant interaction of traumatic load $\times$ genotype $(\mathrm{LR}=5.06, p=32)$.

\section{Discussion}

The results indicate a gene-environment interaction between the human COMT $V \mathrm{Vl}^{158} \mathrm{Met}$ polymorphism and the number of traumatic event types experienced in the risk of developing PTSD. Although Val allele carriers show a typical dose-response relationship between traumatic load and probability of developing PTSD, Met/Met homozygotes show a high risk for PTSD independently of the severity of traumatic load. This finding is in line with previous reports on the effects of the COMT Val ${ }^{158} \mathrm{Met}$ polymorphism: the Met/Met genotype is related to reduced extinction of conditioned fear (11), and the Met allele has been associated with higher reactivity to unpleasant visual stimuli and lower emotional resilience against negative mood states (8) and with higher sensitivity to pain stress (9).

Although it is not possible to draw any causal conclusion from the findings of a genetic association study, we speculate that the underlying mechanisms of the COMT Val ${ }^{158}$ Met polymorphism with regard to PTSD might involve altered dopaminergic and noradrenergic transmission in corticolimbic brain circuitry. Imaging genetics studies have shown that the Met allele, which is associated with lower enzyme activity and thus with higher dopamine levels $(5,6)$, is related to increased brain activity in the ventrolateral prefrontal cortex, hippocampus, and amygdala $(18,34)$. This neural circuitry has been implicated in anxiety and traumatic memory $(35,36)$. Therefore, increased reactivity of brain circuitry implicated in generating and regulating affective responses of COMT Met/Met carriers might underlie their high risk for PTSD even at small traumatic load. For instance, reduced norepinephrine metabolism in Met allele carriers might lead to an increased risk for PTSD after trauma via an increased amygdala fear response, as has been found because of inhibited norepinephrine reuptake (37).

When studying gene-environment interactions, one complication needs to be addressed: does the gene under consideration moderate not only individuals' response to the environment but also- by influencing their behavior-their exposure to environmental risk factors? This would change PTSD prevalence through a different route than enhanced vulnerability after stressors $(38,39)$. However, the present finding that there were no differences in the number of traumatic event types experienced between the genotype groups indicates that the COMT $\mathrm{Val}^{158} \mathrm{Met}$ polymorphism did not lead to altered risk behavior in our sample.

A different common problem in genetic association studies specific to PTSD is that it is unclear how many individuals in a "healthy" (non-PTSD) group might have developed PTSD if they had experienced one or "enough" different traumatic event types $(38,40)$, increasing the danger of false-negative results (41). In this study, the sample consisted of a highly traumatized population, so it was possible to compare persons who had developed PTSD after experiencing traumatic events to severely traumaexposed control subjects without PTSD.

Why was lifetime PTSD related to the COMT genotype,

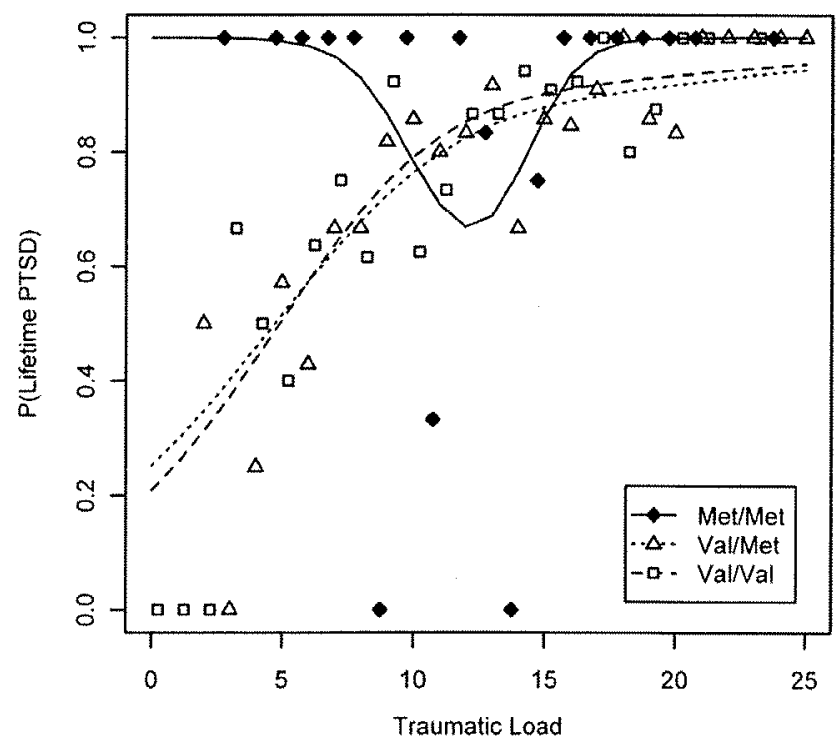

Figure 2. Observed frequencies of participants with lifetime posttraumatic stress disorder (PTSD) (dots) and model fits for lifetime PTSD probability (lines), depending on number of traumatic event types and genotype. Data points for the Met/Met genotype are jittered slightly to the left, whereas data points for the ValNal genotype are jittered slightly to the right. 
whereas current PTSD was not? First we have to consider that a large number of participants with lifetime PTSD remitted spontaneously in the intervening 12 to 13 years between the genocide and our assessment. Furthermore, in a similar and overlapping sample, patients with lower traumatic load were found to have a higher probability of spontaneous remission (Kolassa et al., unpublished observation), which might have erased genotype effects on current PTSD in the present study.

The Met/Met homozygotes were found to be at highest risk of PTSD even after low traumatization, which is in accordance with previous studies that found higher susceptibility to anxiety disorders in Met allele carriers $(10,11,13)$. However, several previous studies also found the Val allele to be associated with higher risk for various psychopathologies and with more abnormality in certain psychopathology-relevant biological measures (15-17,19,23,24). Thus, the role of the COMT Val ${ }^{158}$ Met single nucleotide polymorphism (SNP) in psychopathology might be quite different, depending on the diagnosis (42), which underscores that a given allele cannot be generally considered "better" or "worse" with regard to psychopathology (43).

The allelic frequencies of the COMT Val ${ }^{158}$ Met SNP in our sample of Hutu and Tutsi individuals are very similar to those reported for other sub-Saharan populations like Yoruba, Ibo, Hausa, and Chagga $(44,45)$ (Hapmap project). Although the gross pattern of linkage disequilibrium in the genomic region harboring COMT is similar between Caucasian and African populations, pairwise analyses suggest that the linkage of the $\mathrm{Val}{ }^{158} \mathrm{Met}$ SNP with other potentially functional variants of the COMT gene in intronic and regulatory regions might vary between populations with different ethnic and genetic background. Therefore, future studies with special focus on a dense genomic characterization of the COMT locus in several populations are needed to identify possible main and interacting SNP effects.

In conclusion, subjects homozygous for the Met allele of the COMT Val ${ }^{158} \mathrm{Met}$ polymorphism were found not to be subject to the cumulative effect of PTSD development, where multiple trauma types accumulate to increase the probability of developing PTSD, but to be highly likely to develop PTSD even after very few traumatic event types. This effect is not due to a selection effect of subjects homozygous for the Met allele to have experienced more traumatic events than other participants; therefore it does not seem to be mediated by a higher propensity to experience traumatic situations. Still, it should be kept in mind that the relationship between homozygosity for the Met allele and PTSD is by no means deterministic but rather one of increased susceptibility only, as shown by a number of traumatized Met/Met homozygotes who did not suffer from PTSD.

Limitations to the present study include the small number of Met/Met genotypes in our sample. Furthermore, it was not possible to screen participants for all possible axis I comorbidities, because none of the comprehensive international interviews covering all axis I disorders had been fully tested for reliability and validity in the studied or a comparable population. Future studies should include validated measures of axis I disorders and might elucidate the neural mechanisms that underlie the observed gene-environment interaction with regard to the risk of PTSD

This study was funded by the German Research Fonndation (DFG), grants from the Swiss National Science Foundation to DQ and AP (PPOOP3-123391; CRSIKO_122691; PPOOP3-114813) and from the European Science Foundation (EUROStress), and a stipend of the Zukunftskolleg of the University of Konstanz awarded to ITK. The authors ITK and DQ designed the experiment. The author VE supervised data collection; $A P$ and $D Q$ performed the genetic analyses; ITK and $S K$ perfomed the statistical analyses and wrote the manuscript. All authors commented on and approved the final manuscript. We would like to thank Cindy Eckant and Franka Glöckner for suppont in data collection and Thomas Elbert for valuable suppont in all stages of this study.

All authors reported no biomedical financial interests or potential conficts of interest.

Supplementary material cited in this article is available online.

1. American Psychiatric Association (2000): Diagnostic and Statistical Manual of Mental Disorders (DSM-N-TR). Washington, DC: American Psychatric Association.

2. Neuner $F_{f}$ Schauer $M$, Karunakara $U$, Klaschik $C$, Robert $C$, Elbert $T(2004)$ : Psychological trauma and evidence for enhanced vulnerability for posttraumatic stress disorder through previous trauma among West Nile refugees. BMC Psychiatry 4:34.

3. Kolassa I-T, ErtIV, Kolassa S, Onyut LP, Elbert T (submitted): The probability of spontaneous remission from PISD depends on the number of traumatic event types experienced. Psych Trauma: Theory Res, AndPract.

4. Kolassa I-T, ErtI V, Eckart C, Gloeckner F, Kolassa S, Papassotiropoulos A, et al. (in press): Association study of trauma load and SLC6A4 promoter polymorphism in PTSD: Evidence from survivors of the Rwandan genocide. I Clin Psychiatry.

5. Lachman HM, Pa polos DF, Saito T, Yu M, Szumlanski CL, Weinshilboum RM (1996): Human catechol-O-methyltransferase pharmacogenetics: Description of a functional polymorph ism and its potential application to neuropsychiatric disorders. Pharmacogenetics 6:243-250.

6. Weinshilboum RM, Ottemess DM, Szumlanski CL (1999): Methylation pharmacogenetics: Catechol-O-methyltransferase, thlopurine methyltransferase, and histamine N-methyltransferase. Amnu Rev Pharmacol Toxicol39:19-52.

7. Malhotra AK, Kestler LJ, Mazzanti C, Bates JA, Goldberg $I_{\text {, Goldman D }}$ (2002): A functional polymorphism in the COMT gene and performance on a test of prefrontal cognition. Am J Psychiatry 159:652-654.

8. Heinz A, Smolka MN (2006): The effects of catechol-O-methyitransferase genotype on brain activation elicited by affective stimuli and cognitive tasks. Rev Neurosci 17:359 -367.

9. Xu K, Ernst $M$, Goldman D (2006): Imaging genomics applied to anxiety, stress response, and resiliency. Neuroinformatics 4:51-64.

10. Zubieta J-K, Heitzeg MM, Smith YR, Bueller JA, $X U K_{1} X U Y_{1}$ et al (2003): COMT val ${ }^{158}$ met genotype affects $\mu$-opioid neurotransmitter responses to a pain stressor. Science 299:1240-1243.

11. Lonsdorf TB, Welke Al, Nikamo $P$, Schalling $M$, Hamm AO, Ohman A (2009): Genetic gating of human fear learning and extinction: Possible implications forgene-environment interaction in anxiety disorder. Psycholsci 20:198-206.

12. Montag C, Buckholtz W, Hartmann P, Merz M, Burk C, Hennig J, et al. (2008): COMT genetic variation affects fear processing: Psychophysiological evidence. Behav Neurosci 122:901-909.

13. Enoch $M A, X U K$, Ferro E, Harris CR, Goldman D (2003): Genetic origins of anxiety in women: A role for a functional catechol-O-methyltransferase polymorphism. Psychiat Genet 13:33-41.

14. Ohara K, Nagai M, Suzuki Y (1998): Low activity allele of catechol-Omethytrransferase gene and Japanese unipolar depression. Neuroreport 9:1305-1308.

15. Olsson $C A$, Byrnes $G B$, Anney $R J$, Collins $V$, Hemphill $S A$, Williamson $R$, et al. (2007): COMT Val(158)Met and 5HTTLPR functional loci interact to predict persistence of anxiety across adolescence: Results from the Victorian adolescent health cohort study. Genes Brain Behav 6:647-652.

16. Baekken PM, Skorpen F, Stordal E, Zwart J.A, Hagen K(2008): Depresslon and anxiety in relation to catechol- $O$-methyltransferase Val ${ }^{t 50}$ Met genotype in the general population: The Nord-Trondelag Health Study (HUNT). BMC Psychiatry 8:48. 
17. Craddock $N$, Owen MJ, O'Donovan MC (2006): The catechol-O-methyl transferase (COMD) gene as a candidate for psychiatric phenotypes: Evidence and lessons. Mol Psychiatry 11:446-458.

18. Drabant EMI, Hariri AR, Meyer-Lindenberg A, Munoz KE, Mattay VS, KolachanaBS, et al. (2006): Catechol-O-methyltransferase Val ${ }^{158} \mathrm{Met}$ genotype and neural mechanisms related to affective arousal and regulation. Arch Gen Psychiaty 63:1396-1406.

19. LuBY, Martin KE, Edgar JC, Smith AK, Lewis SF, Escamlla MA, et al. (2007): Effect of catechol-O methyltransferase Val ${ }^{158}$ Met polymorphism on the P50 gating Endophenotype in schizophrenla. Biol Psychiatry 62:822 825 .

20. Barnett JH, Scoriels L, Munafo MR (2008): Meta-analysis of the cognitive effects of the catechol O-methyltransferase gene Val158/108Met polymorphism. Biol Psychiatry 64:137-144.

21. Hosák L (2007): Role of the COMT gene Val ${ }^{158}$ Met polymorphism in mental disorders: A review. Eur Psychiatry 22:276-281.

22. Caspi $A_{\text {s }}$ Moffitt TE (2006): Gene-enviromment interactions in psychia. try: Joining forces with neuroscience. Nat Rev Neurosci 7:583-590.

23. Caspi A, Moffitt TE, Cannon M, MCClay J, Murray $R$, Harrington $H$, et al. (2005): Moderation of the effect of adolescent onset cannabis use on adult psychosis by a functional polymorphism in the catechol-o-methyltransferase gene: Longitudinal evidence of a gene $x$ environment interaction. Biol Psychiatry 57:1117-1127.

24. Thapar $A$, Langley $K$, Fowler $T$, Rice $F$, Turic D, Whittlnger $N$, et al (2005): Catechol-O-methyltransferase gene variant and birth weight predict early-onset antisocial behavior in children with attention-deficit/hyperactivity disorder. Arch Gen Psychiatry 62:1275-1278.

25. Nugent NR, Amstadter AB, Koenen KC (2008): Genetics of post-trau matic stress disorder: Informing clinical conceptualizations and pro moting future research. An I Med Genet C Semin Med Genet 148:127 132 .

26. Foa EB, Cashman L, Jaycox L, Perry $\mathrm{K}(1997)$ : The validation of a selfreport measure of posttraumatic stress disorder: The posttraumatic diagnostic scale. Psycholassess 9:445-451.

27. de Quervain DJ, Kolassa IT, ErtIV, Onyut PL, Neuner F, Elbert T, Papassotiropoulos A (2007): A deletionvariant of the alpha2b-adrenoceptor is related to emotional memory in Europeans and Africans. Nat Neurosd 10:1137-1139.

28. Derogatis LR, Lipman RS, Rickels $K$, Uhlenhuth $E H$, Covi L (1974): The Hopkins symptom checklist: A self-report symptom inventory. BehavSCI $19: 1-15$.

29. Onyut LP, Neuner $F$, Schauer $E$, ErtI V, Odenwald $M$, Schauer $M$, et al. (2004): The Nakivale Camp Mental Health Prolect Building local competency for psychological assistance to traumatised refugees. Interventions 2:90-107.
30. Neuner F, Onyut LP, Ertl V, Odenwald M, Schauer E, Elbert T (2008): Treatment of posttraumatic stress disorder by trained lay counselors in an African refugee settlement: $A$ randomized controlled trial. $J$ Consult Cin Psychol 76:686-694.

31. R Development Core Team (2008): R: A Language and Environment for Statistical Computing. 2.7.0 ed. Vienna, Austria: RFoundation for Statistical Computing.

32. Harrell FE (2001): Regression Modeling Strategies. New York: Springer.

33. Good PI (2005): Permutation, Parametric, and Bootstrop Tests af Hypotheses. New York: Springer.

34. Smolka MN, Schumann G, Wrase J, Grusser SM, Flor $H$, Mann K et at (2005): Catechol-O-methyltransferase Val ${ }^{15}$ Met genotype affects processing of emotional stimuli in the amygdala and prefrontal cortex. INeurosci 25:836-842.

35. Yehuda $R$, LeDoux J (2007): Response variation following trauma: A translational neurosclence approach to understanding PTSD. Neuron $56: 19-32$.

36. McGaugh U (2004): The amygdala modulates the consolidation of memories of emotionally arousing experiences. Annu Rev Neurosci 27 : $1-28$.

37. Onur $\mathrm{OA}$, Walter $\mathrm{H}$, Schaepfer TE, Rehme AK, Schmidt $\mathrm{C}$, Keysers $\mathrm{C}$, et al (2009): Noradrenergic enhancement of amygdala responses to fear. SoC Cogn Affect Neurosci $4: 119-126$.

38. Broekman BF, Olff $M$, Boer $F$ (2007): The genetic background to PTSD. NeurosciBiobehov Rev 31:348-362.

39. True WR, Rice J, Eisen $S A$, Heath $A C$, Goldberg J, Lyons $M J$, et al. (1993): $A$ twin study of genetic and environmental contributions to liability for posttraumatic stress symptoms. Arch Gen Psychiatry 50:257-264.

40. Lee HJ, Lee MS, Kang RH, Kim H, Kim SD, Kee BS, et al. (2005): Influence of the serotonin transporter promoter gene polymorphism on susceptibil. ity to post traumatic stress disorder. Depress Anxiety 21:135-139.

41. Segman RH, Shalev AY (2003): Genetics of posttraumatic stress disorder. CNs Specti 8:693-698.

42. Bilder RM, Volavka I, Lachman HM, Grace AA (2004): The catechol-Omethyltransferase polymorphism: Relations to the tonic phasic dopa. mine hypothesis and neuropsychiatric phenotypes. Neuropsychopharmacology 29:1943-1961.

43. Kendler KS (2005): "A gene for ..." The nature of gene action in psychatric disorders. Am J Psychiatry 162:1243-1252.

44. Palmatier MiA, Kang AM, Kidd KK (1999); Global variation in the frequencles of functionally different catechol-O-methyltransferase alleles. Biof Psychiatry 46:557-567.

45. Palmatier MA, Pakstis AJ, Speed W, Paschou $P$, Goldman D, Odunsi $A$, et al. (2004): COMT haplotypes suggest $P 2$ promoter region relevance for schizophrenia. Mol Psychiatry 9:859-870. 